\title{
Mapas Auto-Organizados (SOM) para la detección de envejecimiento en baterías
}

\author{
P. Pastor Flores ${ }^{1}$, B. Martín-del-Brío ${ }^{2}$, A. Bono Nuez ${ }^{2}$, C. Bernal Ruiz ${ }^{1}$, I. Sanz \\ Gorrachategui $^{1}$ \\ ${ }^{1}$ Group of Power Electronics and Microelectronics (GEPM), \\ ${ }^{2}$ Human Openware Research Lab (HOWLab), \\ Instituto de Investigación en Ingeniería de Aragón (I3A) \\ Universidad de Zaragoza, Mariano Esquillor s/n, 50018, Zaragoza, Spain.
}

Tel. +34-976762707, e-mail: isgorra@unizar.es

\section{Resumen}

Este artículo presenta la problemática asociada a la predicción de degradación de baterías mediante métricas clásicas, proponiéndose una metodología con redes neuronales SOM para clasificar las celdas según condiciones de uso distintas asociadas a diferentes valores de profundidad de descarga, temperatura ambiente y corriente de descarga.

\section{Introducción}

En la actualidad la cantidad de elementos que requieren alimentación autónoma (smartphones, vehículos eléctricos, o instalaciones off-grid) está en constante crecimiento. Ante ese auge, la necesidad de conocer el estado y la forma en la que envejecen las baterías resulta imprescindible. Las baterías se ven degradadas por un gran número de factores, como puede ser la temperatura, la corriente de carga y descarga, el rango de tensiones de trabajo e incluso las diferentes aplicaciones para las cuales son utilizadas.

La métrica más común para definir una batería es su estado de salud $(\mathrm{SoH})$, el cual arroja un porcentaje en función de un cociente definible para varias de las características del modelo como capacidad o impedancia [1]. Sin embargo, esta visión del envejecimiento no tiene en cuenta la no-linealidad del estado de salud. Dos celdas pueden tener el mismo $\mathrm{SoH}$ en ciertos momentos de su vida, pero tratándose de un problema multidimensional, debido a sus diferentes corrientes de descarga, temperaturas o profundidad de descarga, pueden llegar tener una curva de envejecimiento totalmente distinta (Fig. 1).

Se presenta el estudio del envejecimiento de baterías por medio de técnicas de reconocimiento de patrones y análisis exploratorio de datos aplicadas a diversas celdas con distintos usos. La idea de partida es clasificar las celdas en función de la aplicación a la que han servido en sus ciclos pasados, de manera que se pueda dar una predicción de la vida útil remanente

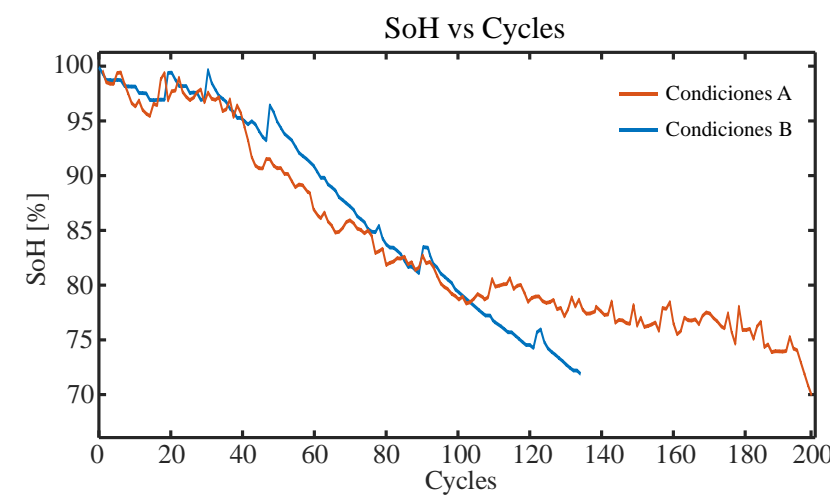

Fig. 1 Comparación SoH en ciclado para dos condiciones distintas

más realista que con otros métodos del estado de la técnica. Para ello, se ha utilizado un algoritmo de clasificación basado en redes neuronales con entrenamiento no supervisado llamado Mapas Autoorganizados (Self-organized Maps, SOM).

\section{Metodología}

Se ha elegido una base de datos creada por la NASA [2], compuesta por 34 celdas de Li-ion de $2 \mathrm{Ah}$, tipo 18650. Los perfiles de carga son iguales para todas las celdas: y los de descarga contienen varias condiciones agrupadas por grupos: variaciones de la corriente de descarga (1,2 y $4 \mathrm{~A})$, temperatura de la cámara climática $\left(4,24\right.$ y $43{ }^{\circ} \mathrm{C}$.) y profundidad de descarga $(2,2.2,2.5$ y $2.7 \mathrm{~V})$.

Como las celdas tienen distintas profundidades de descarga e introducir esta información a la red podría hacer que las agrupaciones realizadas dependiesen de este dato, se "cortan" las curvas de tensión analizadas a un mismo nivel (2.7 V). Para que los vectores de entrada tengan la misma longitud se independizan las curvas de descarga del tiempo y de la capacidad. De esta manera, la red neuronal no podrá recibir ninguna información sobre la duración temporal de las descargas o la capacidad descargada. Esta redimensionalización se ha llevado a cabo interpolando linealmente las curvas de tensión de descarga para conseguir un vector de cien puntos correspondientes a los cien puntos porcentuales del 
estado de carga (SoC). El algoritmo elegido, los mapas autoorganizados (SOM), es un tipo de red neuronal competitiva, con una capa de entrada y una capa de salida (el propio mapa), de entrenamiento no supervisado utilizada para reconocimiento de patrones que genera una proyección no lineal del espacio multidimensional de entrada sobre las dos dimensiones del mapa, permitiendo visualizar la estructura de la base de datos y posibles grupos presentes en ella. Su descripción detallada y funcionamiento se puede encontrar descrito en [3].

\section{Resultados y discusión}

Se estudia validar el algoritmo utilizado para diferenciar celdas empleadas con las características nombradas, reconocidas como cruciales en el estudio del envejecimiento (Fig. 2). Las agrupaciones resultantes tienen resultados claramente polarizados para los mapas de corriente y temperatura. En el caso de las corrientes, se observa un gradiente desde la parte superior izquierda hacia la inferior derecha a través del cual la corriente va disminuyendo. En el caso de la temperatura, aparecen clústers claros para los 4 y $43^{\circ} \mathrm{C}$, sin embargo, para la temperatura de 24 ${ }^{\circ} \mathrm{C}$ aparecen dos polarizaciones distintas. En las agrupaciones de profundidad de descarga se observan coincidencias entre las celdas descargadas a $2.5 \mathrm{~V}$ y $2.7 \mathrm{~V}$ en la zona inferior y $2.0 \mathrm{~V}$ y $2.2 \mathrm{~V}$ en la zona superior del mapa. Sin embargo, estas agrupaciones están claramente supeditadas a las ordenaciones debidas a sus corrientes y temperaturas. Aparece una clara diferenciación en la U-matrix en la zona superior izquierda, coincidente con las celdas descargadas a $4 \mathrm{~A}$. De igual manera se hace presente un subgrupo de las celdas descargadas a 4 A y $43{ }^{\circ} \mathrm{C}$, situadas todas en la zona izquierda-media del mapa, representando las descargas con efectos de degradación más agresivos.

\section{Conclusiones}

En este trabajo se ha estudiado la posibilidad de determinar distintos patrones de uso asociados al envejecimiento en baterías. Al ser este trabajo un estudio inicial, los resultados proporcionados son cualitativos, pero tras el análisis de resultados, se ha comprobado la capacidad de los mapas autoorganizados de discriminar condiciones de temperatura ambiente y corriente de descarga en grupos de celdas con condiciones muy heterogéneas.

Si bien se trata de unos resultados preliminares, las aplicaciones futuras son muy prometedoras. Con el incremento de aplicaciones de alimentación autónoma, la necesidad de determinar el estado de salud real de las baterías va a ser un aspecto crucial en el dimensionado de instalaciones y el diagnóstico de salud para la reutilización de baterías. La distinción de celdas empleadas en aplicaciones diferentes haciendo uso únicamente de los patrones de tensión de descarga de las mismas permitiría clasificar baterías en función de la aplicación a la que han estado destinadas y no únicamente mediante métricas estáticas, como el $\mathrm{SoH}$, que no aportan una información completa de su estado de salud.

\section{REFERENCIAS}

[1] M. Berecibar, I. Gandiaga, I. Villarreal, N. Omar, J. Van Mierlo, y P. Van den Bossche, «Critical review of state of health estimation methods of Li-ion batteries for real applications», Renew. Sustain. Energy Rev., vol. 56, pp. 572-587, abr. 2016.

[2] B. Saha, K. Goebel, y J. Christophersen, «Comparison of prognostic algorithms for estimating remaining useful life of batteries», Trans. Inst. Meas. Control, vol. 31, n. ${ }^{\circ} 3-4$, pp. 293-308, jun. 2009.

[3] T. Kohonen, Self-Organizing Maps, 3. ${ }^{\mathrm{a}}$ ed. SpringerVerlag Berlin Heidelberg, 2001.
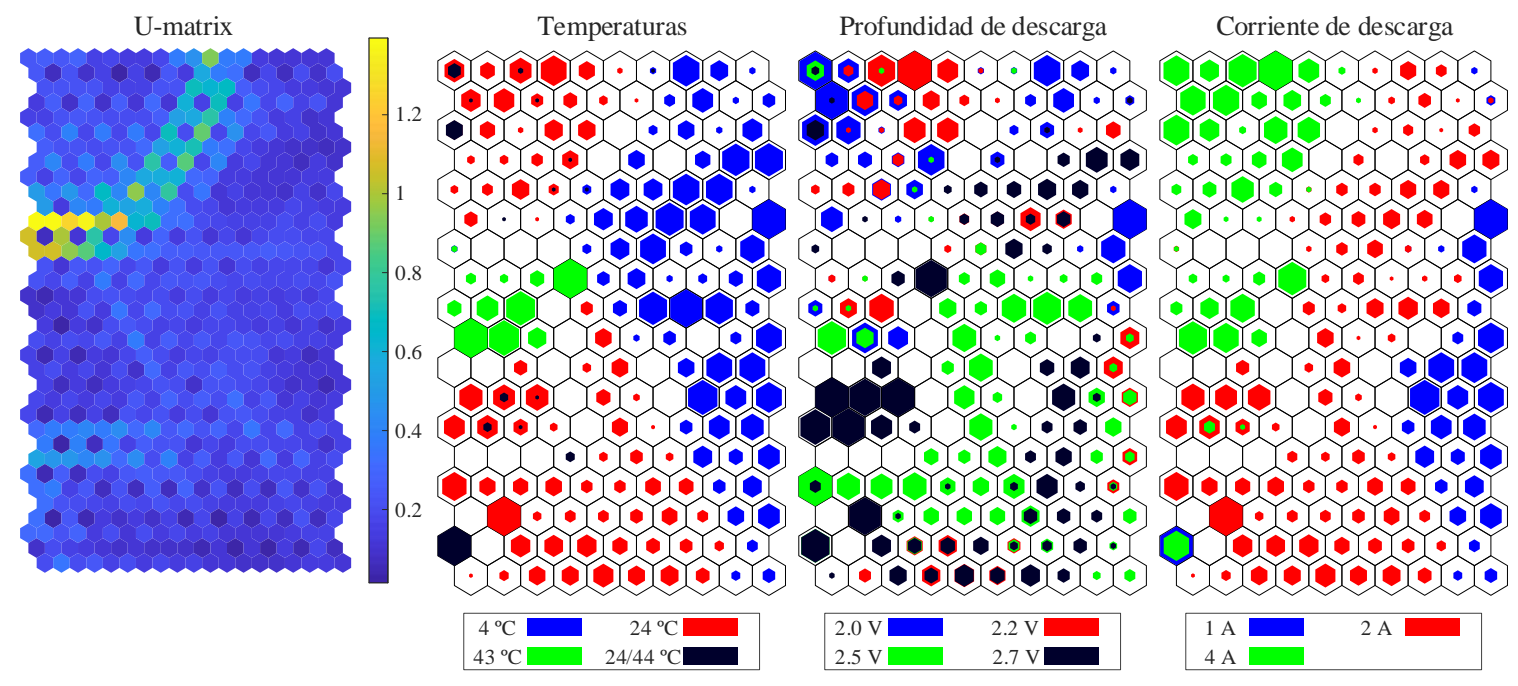

Fig. 2 Resultados del estudio

Revista "Jornada de Jóvenes Investigadores del I3A", vol. 7 (Actas de la VIII Jornada de Jóvenes Investigadores del I3A - 6 de junio de 2019). ISSN 2341-4790. 